\section{Directional geodesic active contour for image segmentation}

\author{
Guopu Zhu, ${ }^{\text {a }}$ Shuqun Zhang, ${ }^{\text {b }}$ Qingshuang Zeng, a and \\ Changhong Wang ${ }^{a}$ \\ ${ }^{a}$ Harbin Institute of Technology, Space Control and \\ Inertial Technology Research Center, Harbin, China \\ E-mail: zgp@hit.edu.cn \\ ${ }^{\mathrm{b}}$ City University of New York, Department of Computer \\ Science, College of Staten Island, Staten Island,
} New York 10314

\begin{abstract}
By incorporating the image gradient directional information into the geodesic active contour model, we propose a novel active contour model called directional geodesic active contour, which has the advantage of selectively detecting the image edges with different gradient directions. The experiment results show the high performance of the proposed active contour in image segmentation, especially when multiple edges with different gradient directions are present near the object boundary to confuse the active contour. (C) 2007 SPIE and IS\&T. [DOI: 10.1117/1.2753836]
\end{abstract}

\section{Introduction}

Since their introduction by Kass et al., ${ }^{1}$ active contours have become very popular for applications in image processing and computer vision. According to the representation of active contours, they can be generally classified into two categories, the explicit active contours ${ }^{1-6}$ represented by point sets or B-splines and the implicit active contours $^{7-13}$ represented by level set functions. The implicit active contours are superior to the explicit active contours in terms of automatically handling topological changes. Most active contours use the magnitude of the image gradient only in image segmentation. Recently, the directional information of the image gradient has been utilized by some explicit active contours ${ }^{3-5}$ to address the issue that the active contour may get confused in image segmentation and converge to the wrong edge when multiple edges with different directions are present near the object boundary. Although some literature ${ }^{11,12}$ on implicit active contours has considered the importance of the gradient directional information, none of them emphasizes the above-mentioned issue. In this paper, based on the original geodesic active contour, ${ }^{9,10}$ which is a typical implicit active contour, a novel active contour model called the directional geodesic active contour is proposed by incorporating the gradient directional information into the geodesic active contour model. Experiments show that the proposed active contour performs better than the geodesic active contour in image segmentation when multiple edges with different image gradient directions are present near the object boundary to confuse the active contour.

Paper 06182LR received Oct. 15, 2006; revised manuscript received Feb. 27, 2007; accepted for publication Apr. 30, 2007; published online Jul. 12, 2007.

1017-9909/2007/16(3)/030501/3/\$25.00 @ 2007 SPIE and IS\&T.

\section{Geodesic Active Contour}

Let $I(x, y): \Omega \rightarrow R^{+}$be a given gray-scale image. The geodesic active contour ${ }^{9,10}$ as an evolving curve is denoted by $C(t)$ and represented by the zero level set of a level set function $\phi(t, x, y)$, i.e., $C(t)=\{(x, y) \mid \phi(t, x, y)=0\}$. The level set function $\phi$ is normally selected to be a signed distance function that is negative in the interior and positive in the exterior of the zero level set. The evolution of the geodesic active contour embedded in the level set function $\phi$ can be formulated by the following partial differential equation:

$$
\frac{\partial \phi}{\partial t}=g(I)\|\nabla \phi\|\left[\operatorname{div}\left(\frac{\nabla \phi}{\|\nabla \phi\|}\right)+\nu\right]+\nabla g(I) \cdot \nabla \phi,
$$

where $\nu$ is a constant parameter controlling the balloon force, and $g$ is the edge indicator function, which is commonly defined by

$g(I)=\frac{1}{1+\left\|\nabla\left(G_{\sigma} * I\right)\right\|^{2}}$,

where $G_{\sigma}$ denotes the Gaussian filter with standard deviation $\sigma$. With an appropriate initialization, the evolving curve $C$ represented by the zero level set of the level set function $\phi$ will stop at the desired edges where the function $g$ is approximated to zero.

\section{Directional Geodesic Active Contour}

Since the geodesic active contour performs the object segmentation essentially by detecting the edges formed at the object boundary, the edge indicator function plays a very important role in the object segmentation. It is seen from Eq. (2) that the edge indicator function $g$ is derived from the image gradient that consists of the gradient magnitude and the gradient direction. However, only the gradient magnitude is used in the function $g$ and the gradient direction is discarded. The function $g$ will be approximated to 0 at the edge location regardless of its corresponding gradient direction, and to 1 in other places. Therefore, it can only detect the presence of an edge but cannot discern the associated gradient direction of the edge. When multiple edges are present near the object boundary, the geodesic active contour that employs the function $g$ may get confused about which is the right edge since all edges have a large gradient magnitude, and may be attracted by the wrong edges instead of the desired object boundary.

In order to prevent the geodesic active contour from being attracted by the wrong edges, we propose incorporating the image gradient direction in the edge indicator function. Here is how we distinguish a desired edge from other undesired edges. An edge is considered desired if its corresponding gradient direction is consistent with the gradient direction of the object edge, i.e., the angle between the two gradient directions is no more than $\pi / 2$. Otherwise, the edge will be considered undesired. Since the object edge is unknown at the beginning, in determining whether an edge is desired, we use the gradient direction of the level set function to replace the gradient direction of the object edge. We can do so because the zero level set of the level set function is generally initialized near the object boundary and will conform to the object boundary ultimately, and the 
object edge's gradient direction will be either identical or opposite to the gradient direction of the level set function $\phi$ when the zero level set superposes on the object boundary. Based on the above analysis, we propose a modified edge indicator function

$k(I, \phi)=\frac{1}{1+d(I, \phi)\left\|\nabla\left(G_{\sigma} * I\right)\right\|^{2}}$,

where the function $d$ is defined according to gradient directions of the image and the level set function and is used to tell if an edge is desired.

For the definition of the function $d$, we choose the convention that the signed distance function for $\phi$ is negative in the interior and positive in the exterior. When the object to be segmented is darker (i.e., has a lower gray value) than the surrounding background, the following function $d$ is used:

$d(I, \phi)=H\left[\nabla\left(G_{\sigma} * I\right) \cdot \nabla \phi\right]$,

where $H(s)$ denotes the Heaviside function, which takes 1 if $s>0$ and 0 if $s<0$. On the contrary, when the object is brighter than the surrounding background, the function $d$ is defined as

$d(I, \phi)=-H\left[\nabla\left(G_{\sigma} * I\right) \cdot \nabla \phi\right]$.

The above definitions of the function $d$ can indicate whether an edge is desired by measuring the angle between the gradient direction of the edge (denoted by $\theta_{\mathrm{E}}$ ) and the gradient direction of the level set function (denoted by $\theta_{\mathrm{L}}$ ). Based on Eq. (4), $d=1$ if $\left|\theta_{\mathrm{E}}-\theta_{\mathrm{L}}\right|<\pi / 2$ (meaning that the edge is desired) and $d=0$ otherwise. Correspondingly, if we substitute the value of $d$ into Eq. (3), the modified edge indicator function $k$ will be approximated to 0 at the location of the desired edge since $d=1$ and the gradient is large, and $k$ will have the value of 1 at those edge locations with undesired directions since $d=0$. Note that the function $k$ is also approximated to 1 in flat areas due to the small gradient. Therefore, if we use the novel edge indicator function $k$ in the geodesic active contour model, the evolving curve will stop at the desired edge only.

Fig. 1 illustrates the difference between the traditional edge indicator function $g$ and the modified edge indicator function $k$ in 1-D format. It can be seen that all four edges are detected by the traditional edge indicator function $g$ regardless of their directions, while only those two edges with the same gradient direction as the level set function are detected by the modified edge indicator function $k$ formulated by Eqs. (3) and (4). Similarly, the modified edge indicator function $k$ formulated by Eqs. (3) and (5) only detects the two edges with opposite gradient direction to that of the level set function. Therefore, the modified edge indicator function can selectively detect the desired image edges.

By replacing the function $g(I)$ in Eq. (1) with the function $k(I, \phi)$, we can obtain a modified geodesic active contour model, referred to as the directional geodesic active contour, with the following evolution equation:
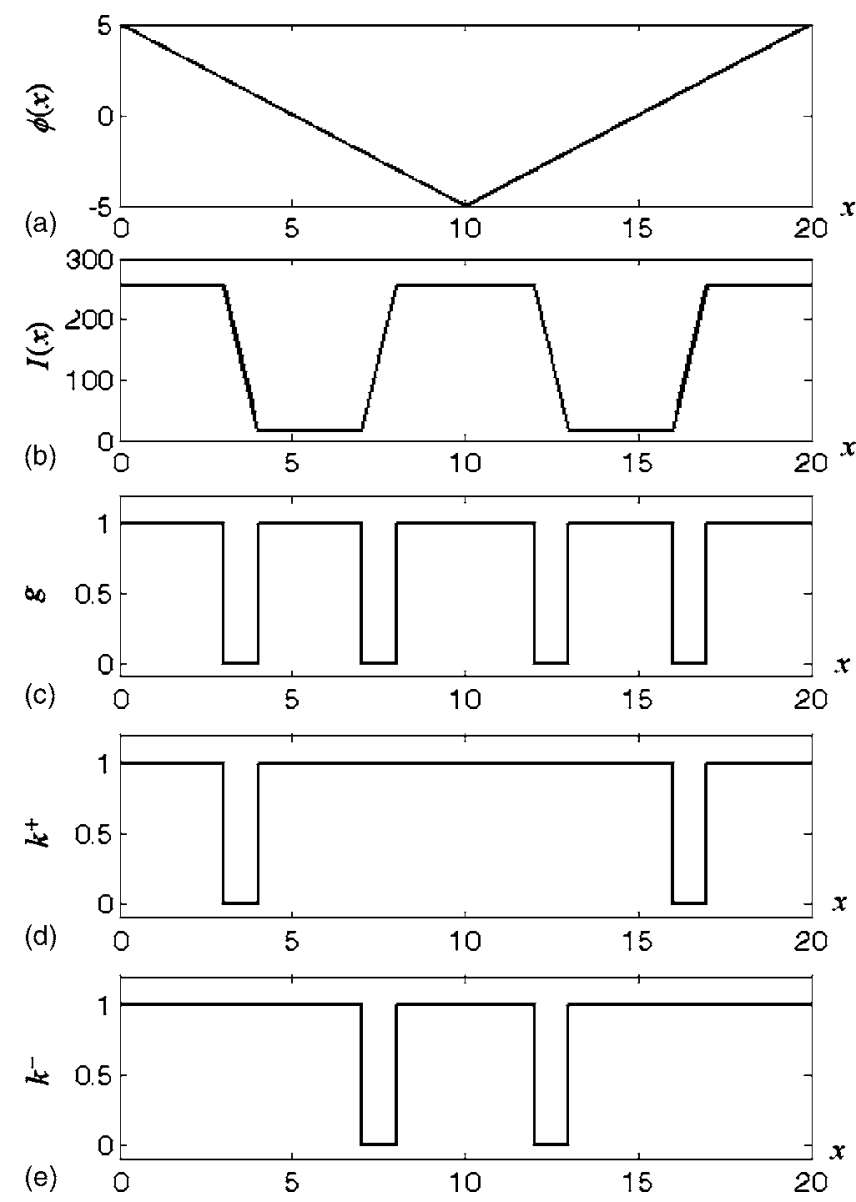

Fig. 1 Comparison between the traditional and the modified edge indicator functions: (a) the level set function $\phi$ at a given time; (b) the image intensity $l$; (c) the traditional edge indicator function $g$; (d) the modified edge indicator function formulated by Eqs. (3) and (4) (denoted by $\mathrm{K}^{+}$); (e) the modified edge indicator function formulated by Eqs. (3) and (5) (denoted by $k$ ).

$\frac{\partial \phi}{\partial t}=k(I, \phi)\|\nabla \phi\|\left[\operatorname{div}\left(\frac{\nabla \phi}{\|\nabla \phi\|}\right)+\nu\right]+\nabla k(I, \phi) \cdot \nabla \phi$.

Based on the property of the edge indicator function $k$, the directional geodesic active contour can avoid being attracted by the edges with undesired gradient directions.

\section{Experimental Results}

Experiments have been conducted on some synthetic and real images to compare the performance of the directional geodesic active contour with that of the traditional geodesic active contour. Here we present two experimental results tested on real $240 \times 240$ images. The upper row of Fig. 2 shows the segmentation of the femur computed tomography (CT) image by the traditional geodesic active contour and the directional geodesic active contour, respectively, under outward balloon force. In this experiment, since the object is brighter than the nearby background, we choose Eqs. (3) and (5) to compute the edge indicator function $k$. The lower row of Fig. 2 shows the segmentation of the lung CT image by the two active contour models, respectively, under inward balloon force. Since the object is darker than the background in this image, Eqs. (3) and (4) are used in 


\section{J E I L E T T E R S}

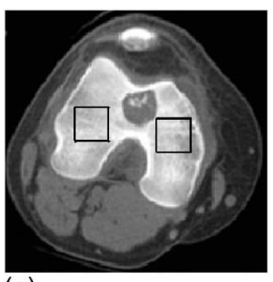

(a)

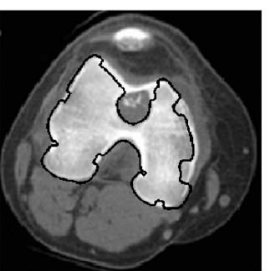

(b)

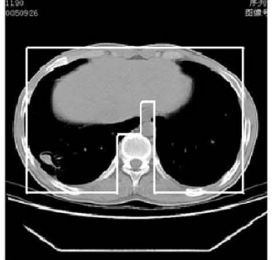

(d)

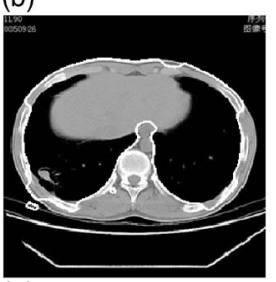

(e)

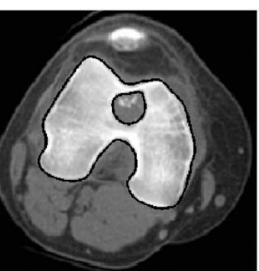

(c)

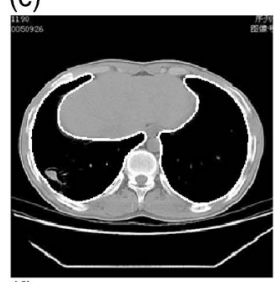

(f)

tect the image edges with different image gradient directions. Experiments conducted on image segmentation show that the directional geodesic active contour is superior to the geodesic active contour when multiple edges with different image gradient directions are present near the object boundary to confuse the active contour.

\section{Acknowledgment}

The authors would like to thank the anonymous reviewers for their valuable comments and suggestions.

\section{References}

1. M. Kass, A. Witkin, and D. Terzopoulos, "Snakes: active contour models," Int. J. Comput. Vis. 1, 321-331 (1988).

2. C. Xu and J. L. Prince, "Snakes, shapes, and gradient vector flow," IEEE Trans. Image Process. 7, 359-369 (1998).

3. H. W. Park, T. Schoepflin, and Y. Kim, "Active contour model with gradient directional information: directional snake," IEEE Trans. Circuits Syst. Video Technol. 11, 252-256 (2001).

Fig. 2 Tests on real images: (a) the original femur CT image with the initial contour; (b) and (c) the segmentation results of the femur $\mathrm{CT}$ image by the traditional geodesic active contour and the directional geodesic active contour, respectively; (d) the original lung CT image with the initial contour; (e) and (f) the segmentation results of the lung CT image by the traditional geodesic active contour and the directional geodesic active contour, respectively.

computing the function $k$. It can be observed from Fig. 2 that in both images there exist some edges with undesired gradient directions near the true object boundary. In both cases, the traditional geodesic active contour is wrongly attracted by the edges with undesired gradient directions, while the directional geodesic active contour can move across the undesired edges and correctly conform to the desired boundaries.

\section{Conclusion}

Based on the geodesic active contour, we have proposed a directional geodesic active contour that can selectively de-

4. M. Jacob, T. Blu, and M. Unser, "Efficient energies and algorithms for parametric snakes," IEEE Trans. Image Process. 13, 1231-1244 (2004).

5. J. Cheng and S. W. Foo, "Dynamic directional gradient vector flow for snakes," IEEE Trans. Image Process. 15, 1563-1571 (2006).

6. G. Zhu, Q. Zeng, and C. Wang, "Simultaneously improving the global and local properties of virtual electric field," Electron. Lett. 42 , 967-968 (2006).

7. V. Caselles, F. Catte, T. Coll, and F. Dibos, "A geometric model for active contours in image processing," Numer. Math. 66, 1-31 (1993).

8. R. Malladi, J. A. Sethian, and B. C. Vemuri, "Shape modeling with front propagation: a level set approach," IEEE Trans. Pattern Anal. Mach. Intell. 17, 158-175 (1995).

9. S. Kichenesamy, A. Kumar, P. Olver, A. Tannenbaum, and A. Yezzi, "Conformal curvature flows: from phase transitions to active contours," Arch. Ration. Mech. Anal. 134, 275-301 (1996).

10. V. Caselles, R. Kimmel, and G. Sapiro, "Geodesic active contours," Int. J. Comput. Vis. 22, 61-79 (1997).

11. A Vasilevskiy and K. Siddiqi, "Flux maximizing geometric flows," IEEE Trans. Pattern Anal. Mach. Intell. 24, 1565-1578 (2002).

12. R. Kimmel and A. M. Bruckstein, "Regularized Laplacian zero crossings as optimal edge integrators," Int. J. Comput. Vis. 53, 225-243 (2003).

13. G. Zhu, Q. Zeng, and C. Wang, "Dual geometric active contour for image segmentation," Opt. Eng. 45, 080505 (2006). 